\title{
Influencing Factors of Eco-Environmental Safety of Mines and Their Green Development: A Case Study of Taoshan Coal Mine in Heilongjiang Province, China
}

\author{
Wei Xiao Gang $\dagger$, Liu Hui Li and Li Guang Hui \\ Department of Civil Engineering and Architecture, Zhengzhou University of Aeronautics, Zhengzhou 450046, China \\ †Corresponding author: Wei Xiao Gang; zzxmwxg@163.com
}

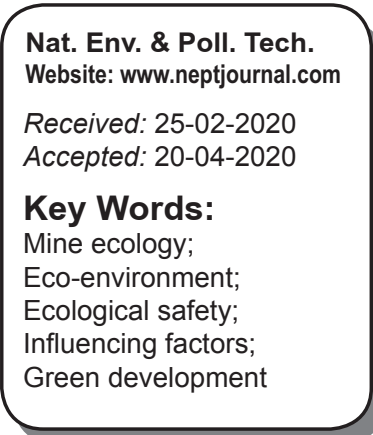

Nat. Env. \& Poll. Tech.

Website: www.neptjournal.com

Received: 25-02-2020

Accepted: 20-04-2020

Key Words:

Mine ecology:

Eco-environment;

Ecological safety;

\begin{abstract}
The demand for mineral resources is continuously increasing due to the accelerated industrialization and urbanization progress. However, most mines in China have backward mining technology, enormous destruction and waste, too low recycling proportion, unstable mineral market, and low management efficiency. Thus, the eco-environmental safety of mines has various difficulties. Identifying the influencing factors of eco-environmental safety of mines, which are typical ecologically vulnerable areas, is important. The green development of mines is a clean production mode that will not generate any harmful effect on human social and natural environments and will be important to realizing sustainable development of mines. In this study, a retrospective analysis of considerable foreign literature on ecoenvironmental safety management of mines in developed countries was conducted. An index system of the influencing factors of eco-environmental safety of Taoshan Coal Mine in Heilongjiang Province, China was established. ISM model (Interpretative Structural Modeling) was used to analyze the influencing factors of eco-environmental safety, and corresponding countermeasures for critical factors were proposed. Results indicate that studies on resource development and utilization and environmental protection of mines in some developed countries, such as America, Australia, and the UK, have been considerably mature. The influencing factors in ISM (Interpretative Structural Modeling) can be divided into three layers, among which the completeness of institution setting for environmental protection supervision is a factor with the most significant influence on mine eco-environmental safety. Input into eco-environmental protection and ecological protection laws and systems of mines are also important factors. The study results are important in determining the most critical influencing factors of mine eco-environmental safety, innovating the new industrial development pattern featured by conservation and intensive and comprehensive utilization of mineral resources, promoting green transformation and upgrading of the mining industry, and boosting sustainable development of the mining industry and eco-environment.
\end{abstract}

\section{INTRODUCTION}

With today's continuous and rapid development of industry-based economy, the increasingly urgent demand for mineral resources has aggravated the imbalance between supply and demand of natural resources; this situation forces enterprises to accelerate resource development. Excessive resource exploitation has caused irretrievable harms despite the enormous economic benefits it brings. Ecosystem degradation, exhaustion of natural resources, and sharp environmental deterioration have far exceeded the load, which can be carried and recovered by the environment. The triggered environmental pollution and ecological damage have hindered economic development to a certain degree. China is starting to enter a rapid development phase of industrialization, urbanization, and marketization; this situation further expands the demand and consumption of mineral resources but hastens the generation of all kinds of small-scale and private mines. The development of mineral resources has endowed enormous economic benefits and abundant material wealth improved the local economic development level and facilitated social progress. However, the unlimited exploitation has led to extreme damage. A large quantity of cultivated lands is also occupied by mining. The local original underground water balance system is destructed as well. Consequently, local people are short of drinking water and experience various geological disasters, such as surface cracking, collapse, and landslide; this situation generates a large number of chemical wastes, damages natural landscapes, and greatly impacts the surrounding environment. Moreover, the environmental quality around original mines has plummeted, and eco-environmental carrying capacity is becoming increasingly fragile. 
Most mines in China, which are traditional resource-based enterprises, have facilitated national economic development. However, the continuously aggravated mine exploitation worsens the environmental situation. Unreasonable mine exploitation has resulted in environmental pollution and damage and frequent geological disasters. Although China has taken related measures and achieved good effects, the severe situation still exists in protecting the geological environment and improving the eco-environment of mines. As shown in Fig. 1, the number of people occupied in mine engineering and its operating revenue in China during 2007-2018 kept a continuous growing tendency with a certain fluctuation. Therefore, extensive exploitation of mineral resources seriously damaged the mine environment, and the surrounding environment failed to evade such impact. The exploitation of mineral resources is accompanied by many secondary disasters, some of which have endangered human living environment. Therefore, analysing the influencing factors of mine ecological safety is important to guarantee a good state of eco-environment needed by survival and development of mining areas and their surrounding ecosystems. In other words, this task does not aim to damage and threaten, mildly if ever, the mining areas as well as the life, health, and settling environment of the surrounding residents and their abilities to adapt to the environment.

\section{PAST STUDIES}

Studies on development and utilization of mineral resources and environmental protection for eco-environmental safety of mines in some developed countries, such as America, Aus- tralia, and the UK, have been quite mature through the longterm adjustment of environmental protection measures for mines. These works have opened and explored a set of paths referable by developing countries and provided many sound strategies and experience in the aspects of eco-environmental safety, green development, and environmental protection of mines. The studies involving eco-environmental safety and green development of mines are presented as follows. Lubchenco et al. deemed that eco-environmental protection should be considered an important objective of mining process and that green and sustainable mine development could be realized only when the geological environment of mines was recovered to the state before mining (Lubchenco et al. 1991). Pring et al. believed that the 21 st century witnessed the industry of mineral resources entering a new era of legal regulation, discussed environmental regulation tendency in mine exploitation process, and analyzed the development status of ever-increasing international influencing laws of various phases from processing to final product in the mining industry (Pring et al. 1999). Weatherstone N. deemed that attention should be paid to the protection of wild animals and water resources in the mining process and investigated the influencing factors of sustainable development opportunity of the mining industry and the way to take these factors as the decision-making basis for sustainable development opportunity (Weatherstone 2005). Maharaj et al. comparatively analyzed the differences between original and newly generated soils in reclaimed land in the aspect of organic carbon and emphasized that organic carbon content in the land during the mining process was the basis for evaluating the quality of reclaimed land in the mining area (Maharaj et al. 2007).

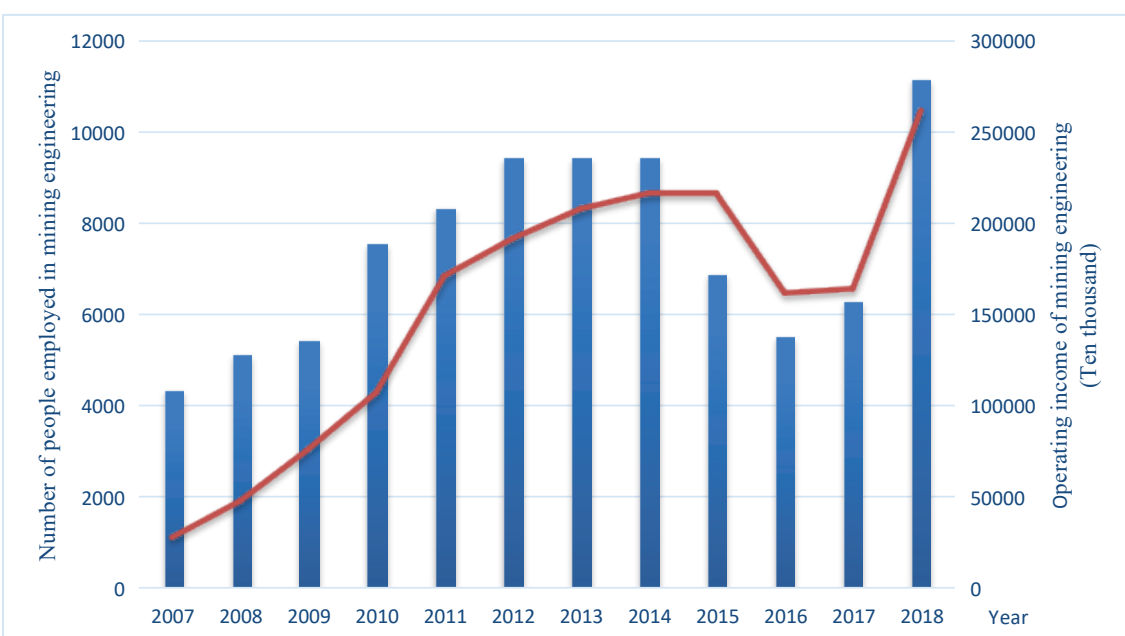

Fig. 1: Number of people occupied in mine engineering and its operation revenue in China during 2007-2018.

(Data derived from National Statistic Database of China (http://data.stats.gov.cn/)). 
Reddick et al. explored the potential of introducing clean production into coal mines and the processing industry in South Africa and stressed that potential clean production would remarkably mitigate the environmental pollution caused by coal mines (Reddick et al. 2008). Wu et al. believed that mine bodies had special geological conditions, backward mining technology, and imperfect management system; all these factors led to mine subsidence in a large area and generated enormous damage of mining environment and infrastructure; these authors also discussed the influence of mining subsidence on eco-environment and public infrastructure and proposed preventive measures (Wu et al. 2009). Franks et al. discussed the reactions made by the mining industry to the sustainable development in the field of waste disposal, introduced a set of sustainable development principles of disposing of mining and mineral processing-induced wastes, and highlighted urgency and necessity of taking new methods in the mining industry (Franks et al. 2011). Tripathi et al. obtained from a survey on age gradients of vegetation on 19-year wasteland in Singrauli mining area in India that soil revegetation in mines was an important management measure of relieving the negative effect of mining and improving the carbon fixation capacity of spoils in mines (Tripathi et al. 2014). Vintró et al. investigated the practice of a small and medium-sized enterprise in the open-pit mining industry in Spain. The results showed that reasonable resource exploitation was a key measure of reducing the environmental pollution. They also introduced some good examples of environmental sustainability (Vintró et al. 2014). Kopacz et al. evaluated the sustainable development level of the hard coal mining industry in Poland. The results indicated that the improvement of sustainable development of the hard coal mining industry in Poland was restricted during the period of analysis. They proved that the green mining industry considerably improved the local environmental status (Kopacz et al. 2017). Lechner et al. deemed that planning of the mining area needed to adapt to the exploitation of mineral products and energy resources and to combine the use of lands already built for agricultural development and ecological protection. They proposed a research framework regarding the development of the mining area for a case study and evaluated the scientificity of this analytical framework (Lechner et al. 2017). Stemn et al. (2019) examined the maturity level of the safety culture of Ghana mines and discussed the relationship between cultural maturity and accident rate. The results showed that the maturity score of the factor safety culture of a mine with low morbidity was always higher than those with high morbidities. The authors proposed improving and intervening fields with weak management of eco-environmental safety of mines. Zuo et al. (2019) deemed that coal mining method in China was already gradually transformed from chamber and pillar method into fully mechanized coal mining. The results showed that surface subsidence in the mining process seriously impacted the geological environment and led to seepage of underground water, decline of water level, and flow of surface water with the ground fracture. Cui et al. (2020) argued that recycling pattern of waste coal resources was the precondition and critical problem for effectively developing idle resources on abandoned mines, particularly Beijing west mining area in China. The results could provide a reference for the recycling of already closed or about to be closed underground coal mines (Cui et al. 2020). Foreign developed countries have achieved a certain performance in environmental protection of mines, which is worthy of reference by mining enterprises and governmental administrative departments in China, through multi-year research and practice. However, few comprehensive evaluation studies on eco-environmental safety of mines are available. Different regions vary in mineral resource endowment, mining technology, technical characteristics, and socio-economic background. Thus, they have greatly different main eco-environmental problems. A comprehensive discussion about their associations remains to be conducted. Meanwhile, different scholars have gaps in professional background and research perspective. Thus, they show regional and subjective features when selecting evaluation indexes and evaluation criteria for eco-environmental safety of mines. Therefore, their evaluation results cannot be compared, which restricts the popularization and application of their evaluation methods and results. Thus, the ISM model was used in the current study to calculate the influencing indexes of eco-environmental safety of a mine in China and its hierarchical structure. In the end, measures promoting green development of the mine were proposed to positively facilitate mining enterprises to take technological innovation as the internal driving force, create a sound macro-environment for reasonably guiding the release of production capacity of mining enterprises for a long time and promoting transformation and upgrading of the mining industry, and provide suggestions for gradually exploring and establishing a long-term mechanism conducive to the green development of the mining industry.

\section{MODEL INTRODUCTION AND INDEX SYSTEM}

\section{Model Introduction (ISM)}

ISM method refers to the interpretative structural modelling method. This method is developed by American Professor J $\mathrm{N}$ to analyze complicated problems related to the socio-economic system. This method decomposes a complicated system into several subsystems (factors) and constructs the system into a multistage hierarchical structural model using 
people's practical experience and knowledge (Warfield et al. 1977). The main calculation steps of the ISM model are as follows:

Analysis of main system factors: Factor analysis is conducted, primary influencing factors of the objects are selected, and the influencing factors needed to construct the ISM model are determined. For the convenience of operation, each influencing factor can be defined as $S_{i}$.

Establishment of the adjacency matrix of influencing factors: On the basis of the overall structure of the influencing factors and their internal correlations, an adjacency matrix $A$ is established, as shown in Formula (1).

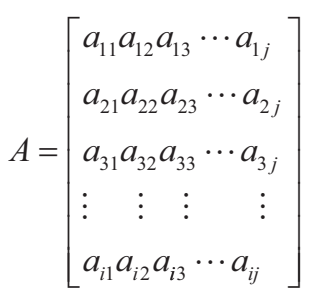

The adjacency matrix $A$ is obtained according to the following principles: if influencing factor $S_{i}$ directly impacts $S_{j}$, then $a_{i j}=1$; otherwise, $a_{i j}=0$. If the influencing factor $S_{j}$ directly influences $S_{i}$, then $a_{i j}=1$; otherwise, $a_{i j}=0$. If strong mutual influence exists between $S_{i}$ and $S_{j}$, then $a_{i j}=a_{j i}=0$.

$M$ Reachable matrix $M$ obtained through calculation: After the adjacency matrix $A$ is acquired, the sum $A+I$ of $A$ and unit matrix $A+I$ is solved, followed by power operation of matrix $A+I$ until Formula (2) holds. Matrix $M$ is called reachable matrix.

$$
M=(A+I)^{r}=(A+I)^{r+1} \neq(A+I)^{r-1}
$$

Decomposition of reachable matrix and establishment of hierarchical structure: The reachable matrix $M$ is decomposed, and reachable set $R\left(S_{i}\right)$, advanced set $A\left(S_{i}\right)$, and the intersection set $R\left(S_{i}\right) \cap A\left(S_{i}\right)$ between the two sets are solved. According to conditions of $R\left(S_{i}\right) \cap A\left(S_{i}\right)=R\left(S_{i}\right), R\left(S_{i}\right)$ is the highest factor set, and $L_{1}, L_{2}, \cdots L_{K}$ denote stages from the top down. $L_{0}=S_{\phi}$ is defined, and $L(n)$ can be obtained using the iterative algorithm, that is, Formula (3).

$$
L_{K}=\left\{S_{i} \in N-L_{0}-L_{1}-\cdots-L_{K-1} \mid R_{K-1}\left(S_{i} \cap A_{K-1}\left(S_{i}\right)=R_{K-1}\left(S_{i}\right)\right\}\right.
$$

On this basis, regional division, inter-stage division, and division of strongly connected blocks can be conducted for the reachable matrix. The row and column corresponding to the highest stage $S_{0}$ are ruled out, and the reachable and advanced sets of the next state are obtained. In the end, the hierarchy chart of the ISM model of each primary factor in the system can be obtained through the above-mentioned analysis, and the model can be further analyzed and explained.

\section{Index System}

By referring to several studies on the influencing factors of mine eco-environmental safety, 18 influencing factors of eco-environmental safety of Taoshan Coal Mine in Heilongjiang Province were concluded. An ISM research group was formed with 14 members composed of administrators of Taoshan Coal Mine, environmental experts, and local residents. The group members comprehensively analyzed the actual situation of eco-environmental safety of Taoshan coal mine. Among the 18 influencing factors, the ISM group believed that eight influencing factors were core influencing factors of Taoshan coal mine, as given in Table 1.

\section{EMPIRICAL RESEARCH}

According to basic conditions of the coal mine, the ISM group combined their own working experience in mines and studies related to the influencing factors of mine eco-environmental safety to obtain the adjacency matrix $A$, as given in Table 2.

According to Formula (2), the reachable matrix $M$, as given in Table 3, can be calculated by Matlab 2012b software.

Table 1: Influencing factors of mine eco-environmental safety.

\begin{tabular}{|lll|}
\hline No. & Factor & Mark \\
\hline 1 & Input into mine eco-environmental protection & $S_{1}$ \\
2 & Institution setting of environmental regulation & $S_{2}$ \\
3 & Improvement of environmental protection methods and means & $S_{3}$ \\
4 & Mine environmental protection access mechanism & $S_{4}$ \\
5 & Environmental restoration margin mechanism & $S_{5}$ \\
6 & Governmental participation in eco-environmental protection & $S_{6}$ \\
7 & Innovation of equipment and process technology & $S_{7}$ \\
\hline
\end{tabular}


Reachable set $R\left(S_{i}\right)$, advanced set $A\left(S_{i}\right)$, and the intersection set $R\left(S_{i}\right) \cap A\left(S_{i}\right)$ between the two are further obtained. Table 4 is obtained according to Formula (3).

Based on the above-mentioned analysis, the ISM model regarding the influencing factors of eco-environmental safety of the coal mine was established, as shown in Fig. 2.

Fig. 2 shows that the institution setting of environmental regulation $\left(S_{2}\right)$ is the most significant influencing factor of eco-environmental safety of Taoshan Coal Mine. Therefore, in most grassroots mine eco-environmental safety management, environmental protection disposition of personnel at all levels of environmental protection regulators influences enterprises' environmental protection methods and means, their environmental protection culture and local participation degree in mine eco-environmental protection, and regulators' thoughts and awareness of environmental protection. This factor especially influences enterprises' cultural construction of environmental protection, shapes concepts, values, and actions in eco-environmental protection during the production and management practice of mining enterprises to a certain degree, and subconsciously influences enterprises' decision-making objectives and employees' behaviours. Input into mine eco-environmental protection $\left(S_{1}\right)$ has a bearing on environmental protection methods and means, equipment renewal, use of advanced production technology, and environmental protection-related cultural construction of enterprises. Moreover, this factor facilitates enterprises to establish their own environmental protection mechanisms through local participation and law enforcement of regulators to reduce eco-environmental pollution and damage of the coal mine from source and strengthen regional eco-environmental safety. Ecological protection laws and systems $\left(S_{8}\right)$ of the mine are the foundation and basis for intensifying environmental management. Environmental regulators, local participants, and responsible departments can do nothing without specific and clear environmental protection laws. Complete laws and systems can improve regulators' ideological understanding, establish complete supervision organizations, and elevate local participation degree in eco-environmental protection to achieve the results obtained through inspection and monitoring of mine eco-environmental safety. Complete laws and systems can also generate a strong promoting effect on environmental access mechanism and environmental restoration margin mechanism for mining to facilitate eco-environmental safety of the coal mine.

Table 2: Adjacency Matrix A.

\begin{tabular}{|lllllllll|}
\hline & $\mathrm{S}_{1}$ & $\mathrm{~S}_{2}$ & $\mathrm{~S}_{3}$ & $\mathrm{~S}_{4}$ & $\mathrm{~S}_{5}$ & $\mathrm{~S}_{6}$ & $\mathrm{~S}_{7}$ & $\mathrm{~S}_{8}$ \\
\hline $\mathrm{S}_{1}$ & 0 & 1 & 0 & 0 & 1 & 1 & 0 & 0 \\
$\mathrm{~S}_{2}$ & 0 & 0 & 0 & 0 & 0 & 0 & 0 & 0 \\
$\mathrm{~S}_{3}$ & 0 & 0 & 0 & 0 & 1 & 0 & 0 & 0 \\
$\mathrm{~S}_{4}$ & 0 & 0 & 1 & 0 & 0 & 1 & 0 & 0 \\
$\mathrm{~S}_{5}$ & 0 & 1 & 1 & 1 & 0 & 0 & 0 & 0 \\
$\mathrm{~S}_{6}$ & 1 & 1 & 0 & 0 & 0 & 0 & 0 & 0 \\
$\mathrm{~S}_{7}$ & 0 & 0 & 1 & 1 & 0 & 0 & 0 & 0 \\
$\mathrm{~S}_{8}$ & 1 & 0 & 0 & 1 & 1 & 1 & 0 & 0 \\
\hline
\end{tabular}

Table 3: Reachable matrix $M$.

\begin{tabular}{|c|c|c|c|c|c|c|c|c|}
\hline & $\mathrm{S}_{1}$ & $\mathrm{~S}_{2}$ & $\mathrm{~S}_{3}$ & $\mathrm{~S}_{4}$ & $\mathrm{~S}_{5}$ & $\mathrm{~S}_{6}$ & $\mathrm{~S}_{7}$ & $\mathrm{~S}_{8}$ \\
\hline $\mathrm{S}_{1}$ & 1 & 1 & 1 & 1 & 1 & 1 & 0 & 0 \\
\hline $\mathrm{S}_{2}$ & 0 & 1 & 0 & 0 & 0 & 0 & 0 & 0 \\
\hline $\mathrm{S}_{3}$ & 1 & 1 & 1 & 1 & 1 & 1 & 0 & 0 \\
\hline $\mathrm{S}_{4}$ & 1 & 1 & 1 & 1 & 1 & 1 & 0 & 0 \\
\hline $\mathrm{S}_{5}$ & 1 & 1 & 1 & 1 & 1 & 1 & 0 & 0 \\
\hline $\mathrm{S}_{6}$ & 1 & 1 & 1 & 1 & 1 & 1 & 0 & 0 \\
\hline $\mathrm{S}_{7}$ & 1 & 1 & 1 & 1 & 1 & 1 & 1 & 0 \\
\hline $\mathrm{S}_{8}$ & 1 & 1 & 1 & 1 & 1 & 1 & 0 & 1 \\
\hline
\end{tabular}




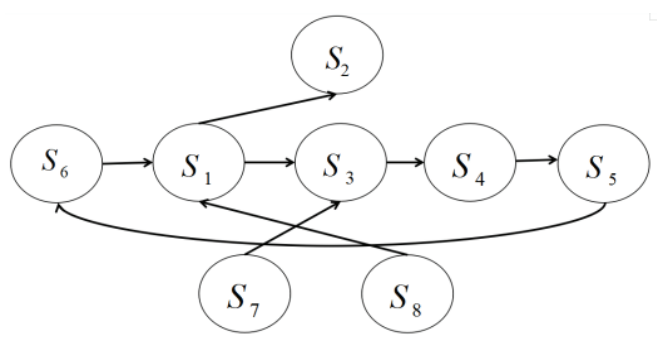

Fig. 2: Three-stage hierarchical ISM model of the influencing factors of eco-environmental safety of Taoshan coal mine.

\section{POLICY SUGGESTIONS FOR GREEN MINE DEVELOPMENT}

\section{Establishment of Long-Term Mechanism for Green Development of the Mining Industry and Reduction in Waste of Resources}

Traditional extensive management should be replaced with intensive management, and mineral resource energy structure should be optimized. Green and clean energy should be energetically developed, the proportion of non-energy sources, such as coalbed gas, natural gas, geotherm, and shale gas should be elevated, $\mathrm{CO}_{2}$ emission should be reduced, local air quality should be improved, and occupation and damage caused by solid wastes to the mining area and surrounding lands should be decreased. Green mining industry-related supporting policies should be comprehensively studied and developed, intensive production and management should be actively encouraged, and rapid development of the mining industry with non-coal clean energy should be facilitated. A long-term mechanism for the development of green mining industry should be reasonably and scientifically constructed, and the development and development pattern coordinating high efficiency and intensiveness should be gradually formed. Special financial funds for package exploration, conservation and comprehensive utilization of mineral resources, and restoration and control of geological environment should be increased, and financing channels should be broadened to enlarge the inclination to green mining enterprises. The government should urge mining enterprises to improve their comprehensive development and utilization level of mineral resources and continuously improve their comprehensive development and utilization modes in accordance with issued construction standards for green mines. The government should also motivate enterprises to undertake social responsibilities, implement energy conservation and emission reduction, and create a good environment for the construction of green mines and the development of the green mining industry.

\section{Broadening of Financing Channels for Mine Environmental Pollution Control and Perfecting Ecological Compensation Mechanism}

The ecological compensation and restoration and environmental government work of mining enterprises should abide by the principle of "give different guidance to different enterprises," that is, different financial support policies and input modes should be implemented for different enterprises. The government should urge mining enterprises to enlarge capital investment and encourage them to construct an ecological compensation mechanism for the sake of vegetation

Table 4: Reachable and advanced sets of the influencing factors of mine ecological safety.

\begin{tabular}{|llll|}
\hline Factor & $R\left(S_{i}\right)$ & $A\left(S_{i}\right)$ & $R\left(S_{i}\right) \cap A\left(S_{i}\right)$ \\
\hline$S_{1}$ & $1,2,3,4,5,6$ & $1,3,4,5,6,7,8$ & $1,3,4,5,6$ \\
$S_{2}$ & 2 & $1,2,3,4,5,6,7,8$ & 2 \\
$S_{3}$ & $1,2,3,4,5,6$ & $1,3,4,5,6,7,8$ & $1,3,4,5,6$ \\
$S_{4}$ & $1,2,3,4,5,6$ & $1,3,4,5,6,7,8$ & $1,3,4,5,6$ \\
$S_{5}$ & $1,2,3,4,5,6$ & $1,3,4,5,6,7,8$ & $1,3,4,5,6$ \\
$S_{6}$ & $1,2,3,4,5,6$ & $1,3,4,5,6,7,8$ & $1,3,4,5,6$ \\
$S_{7}$ & $1,2,3,4,5,6,7$ & 7 & 7 \\
$S_{8}$ & $1,2,3,4,5,6,8$ & 8 & 8 \\
\hline
\end{tabular}


recovery, land reclamation, and environmental protection. In environmental governance and ecological compensation work of mining enterprises, all enterprises should raise funds for restoration and control in addition to national financial supports according to the principle of "whoever develops will be responsible for governance and control." Enterprises should be encouraged to develop and utilize associated minerals and gangues for improving the comprehensive utilization efficiency of resources. Furthermore, they should strictly implement mine eco-environmental governance margin mechanism and facilitate cooperation and coordination among iron mine enterprises, environmental protection departments, and geological department for mineral resources. By deepening integration work of mineral resources, the government can realize optimal allocation of resources. The government should practice industrialization of ecological compensation among iron mine enterprises and guide enterprises' ecological recovery and environmental governance work to a development road of the virtuous cycle.

\section{Accelerating the Development of Green Mining Technology and Reducing Environmental Pollution to the Minimum Extent}

Technological innovation is a driving force for green economic development. The application of green technology can effectively relieve the contradiction between economic development and natural resources and environment and realize the healthy and sustainable development of the mining economy. Associated and secondary resources in the development and utilization of mineral resources can be used to promote the transformation and upgrading of the traditional mining industry, hasten emerging industries, and realize the transition of consumption structure of resources and energy. The development of the green mining industry cannot be separated from a series of technical supports in the exploitation and utilization process of mineral resources, such as green exploration technology of mineral products, clean production technology, environmental monitoring technology, waste reuse technology, pollution abatement technology, and process technology used to prevent pollution. Technological innovation is conducive to the cultivation of emerging industries and improvement and upgrading of the traditional mining industry. Advanced technologies should be used to accelerate the green transformation of geological exploration, which is an advanced concept, culture, and development mode. During the field geological exploration process and under the guidance of win-win balance between green development concept and ore prospecting and environmental protection as the objective while scientific management as the precondition, advanced exploration means, methods, equipment, and processes should be used to reduce negative impacts on eco-environment and restore disturbed eco-environment to the greatest extent.

\section{Reinforcing Law Enforcement Efforts into Mine Environmental Pollution Control and Improving Environmental Awareness}

Law enforcement for mine environmental pollution and environmental protection and pollution prevention should be strengthened. The relationship between environmental law enforcement and environmental protection and environmental pollution prevention should be clearly defined. The government should define the administrative status of law enforcement, complete internal supervision and restriction mechanism in the environmental protection system, establish and perfect environmental early warming and environmental safety network covering the whole province, and realize double protection of mine environment from the angles of system and economy. The emphasis should be laid on the relationship between public and environmental protection. Various environmental protection information should be published to ensure that the public can know enterprises' consciousness of environmental protection and check, suggest, and jointly manage enterprises' environmental protection behaviours. The government should encourage public welfare organizations to unite and make joint efforts in environmental protection and hold down all kinds of ecological destruction activities from legal level. Moreover, the public should be allowed to participate in the establishment of some projects to fully utilize people's strength, protect the environment to the highest degree, and construct a cyclical and green ecosystem. Accordingly, guidance for transforming the requirement for improving the environment into practical environmental protection action can be provided.

\section{CONCLUSION}

Human production activities in the mining industry will not only promote economic development but also directly lead to land ecosystem destruction of the mines. Large-scale and high-intensity resource exploitation of Chinese mines has resulted in a contradiction between supply and demand of resources and ecological problems. The mining process shall not repeat the road of excessive consumption and low-efficiency use of mineral resources. Carrying out the evaluation research of mine ecological safety and proposing the pattern and path of constructing green mines and developing the green mining industry will be important to realizing harmony between mining and eco-environment for establishing a long-term mechanism for the development of the mining industry. A retrospective analysis of studies on mine eco-environmental management in foreign developed 
countries was performed. An index system of the influencing factors of the eco-environmental safety of a mine in China was constructed, and the ISM model was used to analyze the influencing factors of mine eco-environmental safety. The results show that studies on the development and utilization of mineral resources and environmental protection in some developed countries, such as America, Australia, and the UK, have been already quite mature. The completeness of institution setting for environmental regulation is the most significant influencing factor of mine eco-environmental safety among the rest. Input into mine eco-environmental protection and mine ecological protection laws and systems are also important influencing factors. The proposed policy suggestions include establishing a long-term mechanism for the development of green mining industry, broadening the financing channels for mine environmental pollution control, accelerating the development of green mining technology, and strengthening law enforcement for mine environmental pollution. In-depth research should be conducted on exploring new patterns and paths for the development of the green mining industry, enriching the evaluation index systems for mine eco-environmental safety, using combinational evaluation method to give eco-environmental safety rating to one specific mine, and measuring and rating ecological compensation work of mining enterprises.

\section{REFERENCES}

Cui, C.Q., Wang, B., Zhao, Y.X. and Xue, L.M. 2020. Waste mine to emerging wealth: Innovative solutions for abandoned underground coal mine reutilization on a waste management level. Journal of Cleaner Production, 252: 119748.

Franks, D.M., Boger, D.V., Côte, C.M. and Mulligan, D.R. 2011. Sustainable development principles for the disposal of mining and mineral processing wastes. Resources Policy, 36(2): 114-122.

Kopacz, M., Kryzia, D. and Kryzia, K.2017. Assessment of sustainable development of hard coal mining industry in Poland with use of bootstrap sampling and copula-based Monte Carlo simulation. Journal of
Cleaner Production, 159: 359-373.

Lechner, A.M., McIntyre, N., Witt, K., Raymond, C.M., Arnold, S., Scott, M. and Rifkin, W. 2017. Challenges of integrated modelling in mining regions to address social, environmental and economic impacts. Environmental Modelling \& Software, 93: 268-281.

Lubchenco, J., Olson, A.M., Brubaker, L.B., Carpenter, S.R., Holland, M.M., Hubbell, S.P., Levin, S.A., Macmahon, J.A., Matson, P.A. and Melillo, J.M. 1991. The sustainable biosphere initiative: an ecological research agenda: A report from the Ecological Society of America. Ecology, 72(2): 371-412.

Maharaj, S., Barton, C.D., Karathanasis, A.D. and Rowe, H. 2007. Distinguishing" new" from" old" organic carbon in reclaimed coal mine sites using thermogravimetry: II. Field validation. Soil Science, 172(4): 302-312.

Pring, G., Otto, J. and Naito, K. 1999. Trends in international environmental law affecting the minerals industry. Journal of Energy \& Natural Resources Law, 17(1): 39-55.

Reddick, J.F., Blottnitz, H.V. and Kothuis, B. 2008. Cleaner production in the South African coal mining and processing industry: a case study investigation. International Journal of Coal Preparation and Utilization, 28(4): 224-236.

Stemn, E., Bofinger, C., Cliff, D. and Hassall, M. 2019. Examining the relationship between safety culture maturity and safety performance of the mining industry. Safety Science, 113: 345-355.

Tripathi, N., Singh, R.S. and Nathanail, C.P. 2014. Mine spoil acts as a sink of carbon dioxide in Indian dry tropical environment. Science of the Total Environment, 468: 1162-1171.

Vintró, C., Sanmiquel, L. and Freijo, M. 2014. Environmental sustainability in the mining sector: Evidence from Catalan companies. Journal of Cleaner Production, 84: 155-163.

Warfield, J.N. and Fitz, R. 1977. Societal Systems: Planning Policy, Complexity. IEEE Transactions on Systems, Man, and Cybernetics, 7(10): 759-760.

Weatherstone, N. 2005. The role of the mineral reserve estimator in promoting sustainable development in the mining industry. Applied Earth Science, 114(1): 14-22.

Wu, X., Jiang, X.W., Chen, Y.F., Tian, H. and Xu, N.X. 2009. The influences of mining subsidence on the ecological environment and public infrastructure: a case study at the Haolaigou iron ore mine in Baotou, China. Environmental Earth Sciences, 59(4): 803.

Zuo, Q., Wang, Y. and Li, J. 2019. Ground Subsidence in old coal mining area and Its Geological and Ecological Environment Effect. Ekoloji, 28(108): 2429-2433. 